\title{
A MULTI-FIDELITY AERO-THERMAL DESIGN APPROACH FOR SECONDARY AIR SYSTEMS
}

\author{
Dario Amirante \\ Thermo-Fluid System UTC \\ University of Surrey \\ Guildford, Surrey, GU2 7XH, UK \\ Email: d.amirante@surrey.ac.uk \\ Paolo Adami \\ Rolls-Royce Deutschland \\ Eschenweg 11, 15827, Blankenfelde-Mahlow, Germany \\ Email: paolo.adami2@rolls-royce.com
}

Nicholas J. Hills

Thermo-Fluid System UTC

University of Surrey

Guildford, Surrey, GU2 7XH, UK

Email: n.hills@surrey.ac.uk 


\section{ABSTRACT}

The paper presents a multi-disciplinary approach for aero-thermal and heat transfer analysis for internal flows. The versatility and potential benefit offered by the approach is described through the application to a realistic low pressure turbine assembly. The computational method is based on a run time code-coupling architecture that allows mixed models and simulations to be integrated together for the prediction of the sub-system aero-thermal performance. In this specific application the model is consisting of two rotor blades, the embedded vanes, the inter-stage cavity and the solid parts. The geometry represents a real engine situation.

The key element of the approach is the use of a fully modular coupling strategy that aims to combine (1) flexibility for design needs, (2) variable level of modelling for better accuracy and (3) in memory code coupling for preserving computational efficiency in large system and sub-system simulations.

For this particular example Reynolds Averaged Navier-Stokes (RANS) equations are solved for the fluid regions and thermal coupling is enforced with the metal (conjugate heat transfer). Fluid-fluid interfaces use mixing planes between the rotating parts while overlapping regions are exploited to link the cavity flow to the main annulus flow as well as in the cavity itself for mapping of the metal parts and leakages. Metal temperatures predicted by the simulation are compared to those retrieved from a thermal model of the engine, and the results are discussed with reference to the underlying flow physics. 


\section{INTRODUCTION}

In a gas turbine engine, cold air is extracted from the various stages of the compressor and re-introduced in the turbine for cooling and rim sealing. With up to $20 \%$ of air bled off from the main flow [1], the optimization of a secondary air system plays a significant role in improving the overall engine efficiency. In this regard it is key to be able to predict the leakage mass-flow rate, the flow pattern, loss and wall temperature distributions as well as the cavity-main flow interaction including the event of hot gas ingestion inside the cavities. To this aim it is generally accepted that a reliable aero-thermal prediction of these effects in a real system can not be achieved unless adopting coupled cavity-main flow models with real features and accurate models including heat transfer and unsteady effects. Addressing the modelling of these features is not just a challenge about complexity for integration in the industrial practice as a design tool, but more importantly it is a problem about coupling of methods and algorithms (i.e. convergence, stability, different medium properties and scales), use of validated physics and modelling as well as the ability to enable the performance improvements on modern hardware to reduce computational and post-processing costs. From this point of view the secondary air-system is a typical example of a multi-scale and multi-physics problem that is considered in the present paper to describe and demonstrate a coupled modelling approach.

Nowadays CFD is routinely used for secondary air-system design and increasing attention is placed on the predictive capability of the metal temperature. Stand-alone simulations are generally conducted to define the convective boundary conditions which are needed to construct thermal models of the solid. Recent years have also seen a considerable increase in the use of Conjugate Heat Transfer (CHT) analysis with much of this work applied to blade cooling [2,3], pre-swirl systems [4, 5] and disc cavities [6, 7]. With the increase of computational power, aero-thermal studies have gradually moved from single-component analysis to more complex scenarios involving multiple cavities and transient regimes $[8,9,10,11]$.

Despite the recognised improvement achievable with simulations, when considering accuracy, efficiency and reduction of turn-around-time there are still a number of drawbacks that represent challenging hurdles to be addressed. First, heat transfer predictions based on RANS or URANS models suffer from inaccuracies linked to turbulence modelling and are notoriously poor for a variety of flows (most 


\section{Introduction}

noticeably, in the context of secondary air systems, cooling, rim sealing $[12,13]$ and buoyancy induced flows $[14,15])$. Validation and tuning with rig data is therefore required to calibrate predictions and it is fair to state that industrial practice still relies on a certain level of empiricism based on proprietary know-how. Higher order scale resolving methods such as LES $[16,17]$ have the potential to overcome these deficiencies, but are computationally too demanding for day to day use in design. This is particularly the case when trying to address the simulation of interacting components with multi-physics effects since all these effects potentially lead to a wide range of spatial and time scales. A wide-range of scales not only increases the computational cost, but also requires modelling of different flow and thermal regimes that are in many cases ill-conditioning the whole model: if all physical scales in a complex multi-physics model are to be addressed simultaneously, then LES simulations quickly become impractical. Undeniably, the large computational improvements recently achieved have made LES a viable simulation tool in several applications, but its use still continues to pose severe restrictions on the size and complexity for the simulation of integrated multi-scale problems unless clever strategies are put in place.

A second major issue is related to the complexity of geometrical and system integration. An internal cooling system may be considered as composed of a series of sub-systems spread over the entire turbine section, interacting with each other and with the main annulus components. Accounting for these complexities makes the model construction and setup very laborious in particular if special requirements and procedures are to be implemented to maintain the quality and validation of the solution. As more powerful computers increasingly enable larger simulations, other phases of the process (i.e. pre-processing, data management, feature extraction and post-processing) are fast becoming the new bottlenecks of the CFD workflow; these require therefore a design process flexible enough to allow new methodologies for the effective manipulation of heterogeneous large models to be implemented [18].

The work presented in this paper aims to provide a contribution to addressing both points above. In particular it describes a methodology that looks at the multi-scale challenge of aero-thermal simulation from a design point of view, trying to meet requirements of different nature together: (1) modelling accuracy, (2) geometrical complexity/fidelity and (3) flexibility and computing performance. For the purpose of demonstration, a low pressure turbine assembly is considered. The method is based on a 
"divide and conquer" idea that builds on a code coupling framework (JM76) intended for aero-thermal investigations. Instead of addressing the problem as monolithic, fluid and solid regions are divided into smaller sub-domains that are solved by individual sessions of the fluid or thermal solver.

JM76 is based on the use of "smart edges" [19]: dedicated computing resources are deployed for handling the transfer of data between two interacting zones, and generic types of coupling, such as fluidfluid, fluid-solid and solid-solid, are carried out simultaneously. The interfaces between the fluid zones can be treated by means of mixing planes, sliding planes, immersed boundaries or Chimera techniques. The secondary air system is therefore modelled by a modular system consisting of several fluid/solid domains which are interconnected through different instances of the coupler code: these instances are the same number as the interfaces required to connect the individual parts.

Given the aim of delivering a flexible design framework, this coupling logic greatly facilitates the model setup allowing at the same time the replacement of single parts in the complex multi-physics assembly, as well as the use of different methods (i.e. different physical models) during the design iterations. At the same time, it offers a natural split to hybridize the simulation through multi-solver and multi-fidelity coupled sessions, while the costs of the interfaces (typically not scalable) are off-loaded to overlapping instances of the coupling jobs.

The paper is organized as follows: an overview of JM76 is firstly given, along with a short description of the underlying fluid and thermal solvers. A validation example is then presented, for the conjugate heat transfer analysis of a compressor cavity. This is followed by the step-by-step build-up of the low pressure turbine model, serving not only to illustrate the interconnections between the domains, but also to elucidate some of the principles behind the methodology. The results section will analyze the flow field, metal temperature and describe possible strategies for optimizing the computational resources.

\section{COMPUTATIONAL TOOLS}

The work presented is based on Rolls-Royce proprietary software, Hydra and JM76, but the principles and main conclusions from the current demonstration are general. Hydra consists of a suite of fluid modules including a thermal unstructured solver; JM76 is the coupling framework software developed to 
handle interfaces between distinct solvers. For the sake of this application JM76 will be used to couple different parallel instances of Hydra, each one invoking its own solving module. To this purpose Hydra can actually be seen as an external dynamic library object. The next subsections describe briefly the numerical method employed in Hydra and give an overview of the coupling architecture of JM76.

\section{Hydra solver}

Hydra is an unstructured finite-volume solver of the compressible Reynolds Averaged Navier-Stokes equations. The code is vertex-centered, and the "median-dual" constructed around each mesh node defines the local control volume [20].

The spatial discretization is performed using the approximate Riemann solver of Roe [21] for the convective fluxes. For the approximation of the viscous fluxes, flow variables gradients are evaluated at the mesh nodes using a Green-Gauss method and a consistent finite-volume scheme is obtained by averaging the gradients at the interfaces between two adjacent control volumes. A formal second-order of accuracy of the spatial discretization is achieved by modifying the upwind contribution of the Roe dissipative flux with the introduction of pseudo-laplacian operators, as detailed in the work of Moinier [20]. In steady RANS computations, the flow equations are iterated using a Runge-Kutta (RK) $m$-stage method:

$$
\mathbf{W}^{(k)}=\mathbf{W}^{(0)}-\frac{\alpha^{(k)}}{V} \Delta t \mathbf{R}\left(\mathbf{W}^{(k-1)}\right) \quad k=1 . . m
$$

In Eqn. $1, \mathbf{W}$ is the vector of conservative variables, $V$ is the control volume, $\mathbf{R}$ is the residual function deriving from the spatial discretization, $\Delta t$ is the time step and $\alpha^{(k)}$ are the RK coefficients. Two choices to accelerate convergence are considered in this paper. In the default option, a 5-stage RK scheme (RK5) is used as the smoother in an edge-collapsing multigrid algorithm [20]. Alternatively, a semi-implicit variant for the 3-stage RK scheme (RK3) is obtained using an implicit preconditioner and executed on a single grid [22]. In this case, the residual function $\mathbf{R}$ on the right hand side of Eqn. 1 is premultiplied by a preconditioner matrix $\mathbf{P}^{-\mathbf{1}}$. Following the formulation of Swanson et al. [23], $\mathbf{P}$ is given by the Jacobian of an approximate residual function constructed with first-order spatial discretizations. The 
implicit preconditioner requires at each stage of the RK3 the solution of the linear system

$$
\mathbf{P} \tilde{\mathbf{R}}=\mathbf{R}
$$

with

$$
\mathbf{P} \simeq \frac{\partial \mathbf{R}}{\partial \mathbf{W}}
$$

The linear system is solved using the Richardson method with incomplete lower-upper (ILU-0) preconditioning. Since the role of $\mathbf{P}^{-\mathbf{1}}$ is to reduce the stiffness of the discrete equations, there is no need to obtain an accurate solution, and only a few Richardson iterations $(\leq 10)$ are performed.

The thermal module in Hydra inherits most of the features from the explicit flow solver. The unsteady heat conduction equation is discretized in a finite-volume approach, and integrated in time using an identical time-marching scheme. Simplified versions of the same subroutines are used to approximate the temperature gradients at the grid nodes, and to compute the viscous fluxes needed for the evaluation of the residuals. The semi-implicit RK method is currently not available for the thermal solver.

\section{Coupling code}

A schematic representation of a generic coupled model in JM76 is shown in Fig. 1. The whole system is decomposed as a number of Hydra Sessions (HS) and Coupler Units (CU). The Hydra Sessions are distinct jobs as well as models (i.e. solid or fluid) covering adjacent or overlapping computational domains. Coupler Units are distinct sets of computing processes dedicated to transfer information between the Hydra Sessions and tasked with performing the required interpolations at the interfaces.

In JM76, an interface is a set of points through which two individual jobs/models communicate. In the simplest case, the points identify adjacent surfaces from the CAD model, but, more generally, they can be a portion of volume or any sort of artificial boundary identified inside the system.

The Coupler Unit of JM76 implements the concept of "smart edge" [19], also referred to as "smart interface methodology" by some authors [24]. It is essentially a program that follows a sequence of instructions on a two-entry data set pertaining to a specific interface. Thus, a Coupler Unit connects two (and only two) sessions by handling the corresponding interface. Each individual interface between two 


\section{Validation}

sessions does require a dedicated Coupler Unit: as it happens between HS3 and HS4 in the example of Fig. 1.

The sequence of operations that a Coupler Unit performs on its own interface depends on an attribute specifying the interface type. For example, interfaces defined as sliding planes require search operations whereas circumferential averages are performed for interfaces defined as mixing planes. The Coupler Unit replaces what in CHIMPS [25] or OPALM [16] is the driver program responsible for the algorithmic coupling procedure. The fundamental difference is that a Coupler Unit is limited to the interface between two connected models. In this context, the JM76 architecture may be considered halfway between coupling frameworks based on communication hubs (CHIMPS [25], FlowSimulator [26]) and those built on direct communications between codes (preCISE [27], UMI [28]).

A Coupler Unit defines the corresponding interface using an unstructured mesh format. When the program is launched, all Hydra Sessions carry out autonomously their internal partitioning. Afterwards, each Coupler Unit gathers from the processes responsible for the two attached Hydra Sessions lists with nodes coordinates and mesh elements. The Coupler Unit assembles these data constructing the two meshes (one for each attached HS) that define the interface.

The Hydra processes follow the operations described in Alg. 1: there is an external loop over the number of iterations to perform (ncycle), an intermediate loop representing a multigrid cycle, and an internal loop over the Runge-Kutta stages. For the explicit scheme, a multigrid cycle consists of applying repeatedly the RK5 scheme on various grid levels. For the semi-implicit scheme, a cycle corresponds to a number of Richardson iterations performed within the RK3 scheme on the finest grid. The Hydra processes parse the coupled interfaces for each external iteration, and send data to the connected CU according to a coupling frequency specified independently for each interface. The strength of the coupling between two models can be controlled by changing the coupling frequency or by modifying the definition of a single cycle (i.e. number of grid levels, number of Runge-Kutta calls per grid level and number of Richardson iterations). It will be shown how this feature may have a significant impact on convergence speed. In a coupled system where different flow and thermal regimes coexist, the computational resources can be allocated according to the physical scale of each sub-system. Load balancing can then be preserved 
by adjusting one of the parameters discussed above.

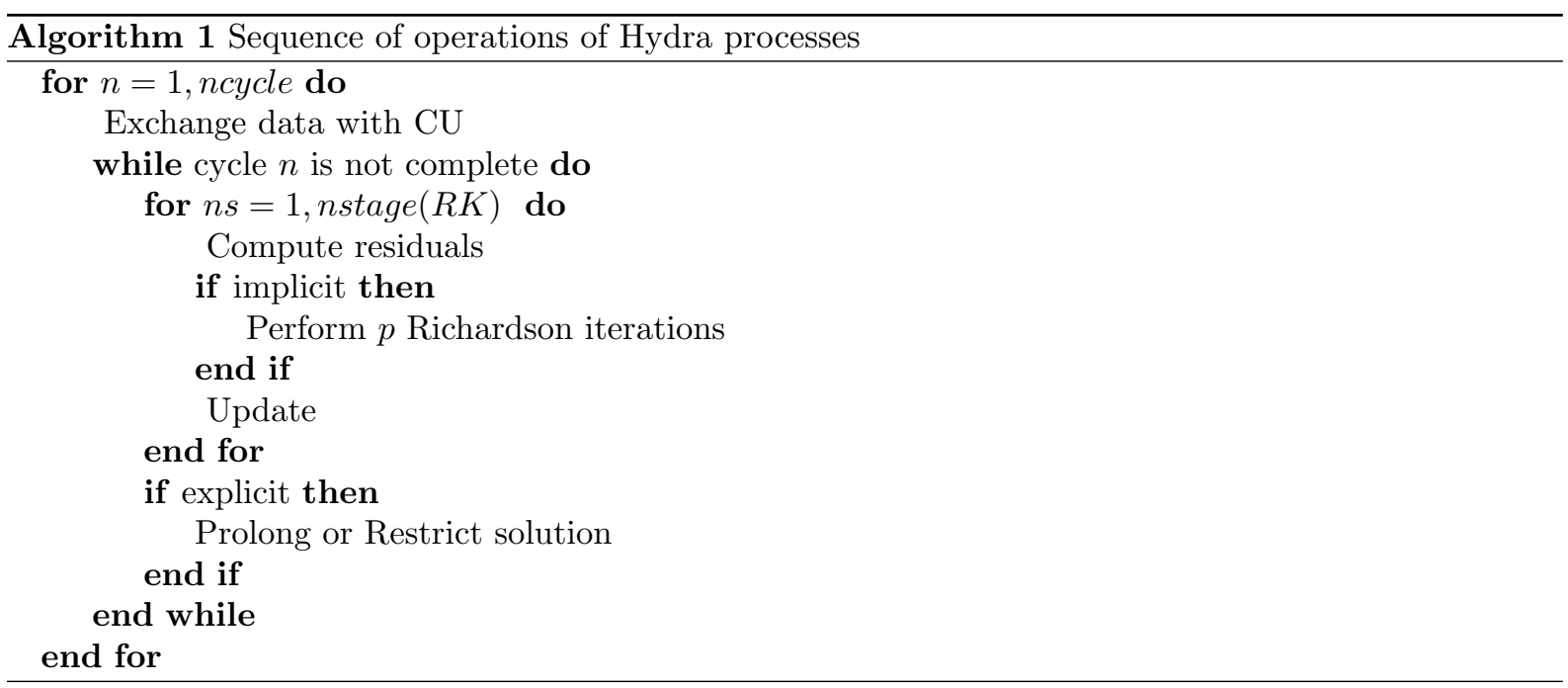

\section{VALIDATION}

Validation of the methodology has been conducted for the conjugate analysis of a test rig modeling a compressor cavity. The test rig is shown in Fig. 2 and consists of a stationary shaft located at inner radius (coloured in grey), an external casing (medium grey) and four co-rotating cavities separated by three discs (light grey).

The rotating cavities are heated on the outer surface by an external heater (not shown in the figure) located between the rotor and the external casing. An axial throughflow of cooling air (the bore flow) is introduced in the annular gap between the discs and the shaft, and a small amount of air is introduced from the shroud in the two intermediate cavities.

Figure 3 shows the JM76 model adopted for the test rig. There are five Hydra Sessions corresponding to one solid model for the entire rotor, and one fluid model for each cavity. The decomposition of the fluid domain is shown in Fig. 4. The interfaces between the cavities are mixing planes located below each disc. The communications between fluid and solid models occur through four separate CHT interfaces.

All models have a rotational periodicity of 90 degrees and the meshes consist of 1 million cells for the solid region, 2 million cells for the enclosed cavities (CAV1 and CAV4) and 16 million cells for CAV2 and CAV3. This resolution was the result of a grid convergence study focused on the temperature of 
DISC3, where the majority of the thermocouples are located (Fig. 2). The computational resources are distributed among the various Hydra Sessions accordingly and only one process is used for each Coupler Unit. The $k-\omega S S T$ turbulence model is adopted for all the fluid zones.

Table 1 gives the details of the running conditions for the rig. These include the rotor angular speed $\Omega$ and the relevant inlet temperatures of the fluid. The metal temperature prescribed on the outer surface of the rotor is reported in Fig. 5. The rotational Reynolds number and the axial Reynolds number of the bore flow are representative of engine conditions [29]. The mass flow ratio between radial inflow and axial throughflow is equal to $4.4 \%$ of the throughflow and somewhat higher than the values expected in a real engine.

Figure 5 shows the metal temperature predicted along the internal surfaces of the cavities. The blue symbols identify the temperature prescribed along the outer surface of the rotor. There is a substantial agreement with the measurements, especially for the two intermediate cavities. For a proper interpretation of the results, it is important to consider the qualitative behaviour of the flow. Temperature contour plots are shown in figure 6. It can be noticed that separate color bands are aligned almost horizontally, and extend from the fluid domain into the solid domain. This indicates a stratified core within the two intermediate cavities, and almost adiabatic conditions on the surface of DISC3. The behaviour observed in CAV1 and CAV4 is very different. At a given radial position, the fluid is significantly colder than the metal. This is due to the presence of cold fluid that penetrates from the bore flow into the cavities. For the limited number of points available on DISC2 and DISC4, the agreement between data and computations is less satisfactory. However, considering that buoyancy induced flow may be affected by the sector size of the CFD model [14] and in light of the marginal resolution employed for CAV1 and CAV4, the overall result of the simulation is deemed very good.

\section{LOW PRESSURE TURBINE}

Figure 7 shows the secondary air system in a low pressure turbine of an existing engine. The cooling flow extracted from the compressor is collected into the large inner cavity, and enters the outer inter-stage cavities through specific holes, identified by red arrows in Fig. 7. Using the current approach, the analysis 
can be easily extended by adding more parts to cover the entire assembly. In this paper, with the aim of demonstration, we focus the attention on the stator well enclosed in the square.

To facilitate the description of the model, it is convenient to follow the set-up of the various components in steps. Figure 8 shows the fluid zones adopted for the main annulus section. These consist of two stators (LOS1, LOS2) and one rotor (LOR1) blade, separated by two interfaces defined to be of type mixing planes. This is a standard interface used between stationary and rotating rows in steady RANS; although not shown in this work, unsteady interaction is also allowed and in this case the interface should be set to type sliding plane. The fluid zone LOS1 includes only one vane and has a rotational periodicity of $3.6^{\circ}$, whereas both LOR1 and LOS2 are sectors of 18 degrees with a blade count of 5 . In light of a planned extension to Unsteady RANS, this choice represents a good compromise between accuracy and computational cost. As previously mentioned, in JM76 each domain corresponds to an individual Hydra model, and each interface is hold by a Coupler Unit. Up to this point the partial system includes therefore 3 Hydra Sessions (steady) and 2 Coupler Units.

The stator well under investigation lies below the vanes of LOS2, and is labelled as CAV1 in Fig. 9. The interface between LOS2 and CAV1 is of type "overset", and includes two couples of immersed boundaries (IMB) located in the regions of the front and rear seals. Immersed boundaries are defined as boundary groups that fall within the mesh of an overlapping domain. Using the overset (or Chimera) methodology $[30,31]$, the two solutions in LOS2 and CAV1 are joined by interpolating the flow field on the IMB nodes from the solution of the overlapping mesh.

The fluid zone CAV1 has been meshed as hollow. The stator disc is incorporated through the use of an additional interface of type overset, connecting CAV1 to a fluid zone constructed around the stator disc. This is denoted by BL-DISC in Fig. 10. The mesh of zone BL-DISC is fully embedded into that of CAV1. An hole-cutting technique identifies the nodes of CAV1 that fall within the domain of BLDISC. These nodes form a hole and, using Chimera terminology, are blanked out during the computation. Mesh nodes of CAV1 that surround the hole form an artificial boundary ("the fringe") where the flow variables are interpolated using the solution in the model BL-DISC. Similarly, the fluid model BL-DISC includes boundary nodes immersed into CAV1. Hence, the solution in CAV1 is used to interpolate the 
flow variables onto the IMB nodes of BL-DISC.

Figure 11 shows the hole region derived in CAV1 using the surface normal vector method [31]. The fringe nodes form an artificial boundary of irregular shape, located at a short distance (controlled by the user) from the immersed boundaries of BL-DISC. The hole-cutting, and the subsequent search operations to find donor cells in the overlapping mesh are performed on the fly by the Hydra-JM76 processes. The user only needs to specify in the JM76 input file which boundary group serves as IMB boundary or as cutting wall for the coupled domain.

The Chimera method has also been adopted to model the inner cavity, as shown in Fig. 12. Here, a background mesh (CAV2) contains a body-fitted mesh (BL-CAV2) which incorporates all the irregular features at the borders of the cavity. In this case the hole nodes excluded from the computations are those of CAV2 that lie outside the inner borders of BL-CAV2. Clearly, this approach simplifies meshing, helps improve grid quality and is particularly suitable for parametric studies on the geometric characteristic of a single component that can be easily replaced in the assembly without the need to reprocess the entire model. Moreover, the overset/Chimera method nestled inside JM76, constitutes a natural infrastructure for multi-fidelity simulations of complex systems. In fact, the setting of the CFD method in the various Hydra Sessions can be heterogeneous: this means that different discretization options or schemes as well as differently tuned turbulence models can be used in each part as appropriate.

Four distinct Hydra Sessions are considered for the solid models (Fig. 13), each with its own thermal conductivity. The solid surfaces surrounded by the flow are coupled with the appropriate fluid zone through interfaces of type "Conjugate Heat Transfer" (CHT). Following a common procedure $[4,5,16]$, thermal coupling on CHT interfaces is achieved applying wall temperature on the fluid side, and heat flux on the solid side. The interconnection between distinct solids in contact occurs via interface of type "Thermal Contact" (THC). In our model these are located at the root of the blades and vanes. Formally, THC interfaces are treated in the same way as CHT interfaces, i.e. by exchanging temperature and heat flux, but in some circumstances (not considered in this paper) they can include a thermal contact resistance between the materials. The final complete JM76 model includes 11 Hydra Sessions, 14 Coupler Units and is schematized in Fig. 14. The logic of the interconnections can be easily recognized in light 
of the description just given. For example, LOS2 is coupled through a mixing plane (MIX) to LOR1, an overset interface (OSET) to the cavity flow in CAV1, a CHT interface to the solid model of the disc, and a CHT interface to the solid model of the vane.

A final consideration is important as conclusion of the model set-up. From the perspective of a Coupler Unit, an overset interface is interpreted in the same way as any other type of interface, namely as a couple of unstructured meshes. The task of identifying the points forming these meshes falls on the Hydra-JM76 processes and is performed on the basis of a rule associated with the interface type. This could be a boundary group membership, a fringe membership or any other sort of criterion.

\section{MESH AND BOUNDARY CONDITIONS}

With the arrangement described in previous section, the boundary conditions remain to be specified in a few locations of the system. In the main annulus, inlet total pressure and total temperatures are prescribed at the entrance of LOS1 whereas a static pressure with enforced radial equilibrium is fixed at the outlet of LOS2. A total temperature and a fixed mass flow rate are imposed for the coolant inlet of CAV1, in agreement with data retrieved from a thermal model of the engine.

The inflow specification of the coolant entering the inner cavity, CAV2, is more problematic. This flow, in fact, branches out for all the outer cavities, even those which are not included in the JM76 model. For this reason, it has been decided to assume the same flow rate specified at the entrance of CAV1. The majority of the wall boundaries of the solid models receive heat flux from the coupled fluid zones. An exception is made for the two outer surfaces in the rear of SOLID-CAV, adjacent to the next turbine stage. For these surfaces, a wall temperature was prescribed using data from the thermal model.

Table 2 gives the mesh size of each model. All the meshes consist of hexahedral elements except the solid models of blades and vanes which are made of tetrahedra. Near wall grid spacing for fluid meshes was adjusted to have $y^{+} \leq 10$ for the cavity flows, and slightly higher value $\left(y^{+} \leq 15\right)$ for the annulus flow. The $k-\omega S S T$ was used as turbulence model. At the moment JM76 does not incorporate any method for automatic allocation of the computational resources. These are prescribed by the user and, as will be shown later, can be conveniently calibrated judging by the complexity of each model. 
Coupling frequency was set equal to 1 cycle for all fluid-fluid interfaces and to 200 cycles for CHT and THC interfaces. For this choice, we relied on best practice acquired from previous simpler models.

\section{RESULTS}

The section is divided in three parts. First, we describe the flow physics. Then, metal temperatures are compared with those retrieved from a thermal model of the engine based on heat transfer correlations and validated by engine testing. The last part will focus on optimizing the computational resources.

\section{Flow physics}

Figure 15 shows a contour plot of the fluid temperature on the periodic surface of the stator well model. Two-dimensional streamlines of the flow are also reported. The temperature is nondimensionalized using the total temperature $T_{0}$ specified at the entry of LOS1. It can be seen that the coolant injected is divided in two parts. Some of it moves through the labyrinth seal and enters the rear cavity. The remainder merges with the main annulus flow moving through the front rim seal. This is the qualitative behaviour expected when the coolant supply exceeds the labyrinth demand, and the cavity is not perfectly balanced. However, the temperature level in the rear cavity is significantly lower, indicating that the leakage flow sucked into the labyrinth seal is larger than the flow moving through the rim seal.

Figure 16 shows the swirl ratio computed in the inner cavity. All the boundaries of the cavity rotate, except the one on the left wall. Hence, the flow configuration resembles that of a rotor-stator cavity with radial outflow [32]. The coolant is driven outward along the lower rotating wall, before being diverted towards the cavity where the outlet is. It should be noted in both Fig. 15 and Fig. 16, the smooth behaviour of the contour bands across the overset interfaces, indicating the consistency of the solutions on the overlapping meshes.

Coupled simulations may sometimes suffer from poor convergence or instability, and is therefore important to examine this aspect. Figures 17 and 18 report the convergence histories pertaining to each model. Fluid models LOS1, LOR1, CAV2 and BL-CAV2 show a clear decreasing trend. In CAV1, BLDISC and LOS2, after an initial drop of almost three orders of magnitudes, occurring during the first 10 thousand cycles, the curves flatten around a similar value (Fig. 17). A closer examination of the log 
files revealed that the nodes with maximum residual values changed randomly, but were always located in the front rim seal region. This behaviour results from natural unsteadiness of the flow. Figure 19 shows a contour plot of axial velocity on a cross-section cutting the domain of CAV1 at the axial position where the immersed boundary of LOS2 is located. The velocity is nondimensionalized by the annulus velocity $U_{i}$. Contour levels have been restricted to $10 \%$ of $U_{i}$ to highlight a non-uniform circumferential distribution of the velocity field, with regions where the flow leaves the cavity to enter the annulus, and regions where the annulus gas is ingested into the cavity.

The convergence histories for the solid models is significantly slower (Fig. 18), and seems to reflect the unsteadiness of the fluid zones. It can be noticed, in fact, that the residuals in SOLID-DISC and SOLID-CAV drop between two communication points (the frequency exchange is set equal to 200 cycles) and then, once the heat fluxes are updated, return to their previous values.

Table 3 reports mass flow rates predicted at various interfaces of the model. All values are nondimensionalized by $\dot{m}_{i}$, i.e. the mass flow rate at the entry of LOS1. The mass imbalance between LOS2 inlet $\left(\dot{m}_{i}^{S 2}\right)$, LOS2 outlet $\left(\dot{m}_{o}^{S 2}\right)$ and secondary flow $\left(\dot{m}_{c}\right)$ is given by $\dot{m}_{o}^{S 2}-\left(\dot{m}_{i}^{S 2}+\dot{m}_{c}\right)$ and corresponds to $1.4 \%$ of the coolant $\dot{m}_{c}$, and to $0.012 \%$ of $\dot{m}_{i}^{S 2}$. Mass imbalance between LOS1 and LOS2 inlets $\left(\dot{m}_{i}^{S 2}-\dot{m}_{i}\right)$ corresponds to $0.62 \%$ of the annulus flow $\dot{m}_{i}$. Finally, Figure 20 shows the evolution of mass flow rate through the front rim seal, measured from both sides of the overset interface between LOS2 and CAV1. It can be seen that the two values are virtually the same, and fluctuate around $0.158 \%$ of the annulus flow.

\section{Metal temperatures}

Figure 21 and 22 show the computed metal temperatures. Comparisons with the solution of the thermal model, hereafter referred to as SC03 solution, are given in Fig. 23, 24 and 25, for a set of control points. The exact location of the control points is specified in Fig. 22.

For a better interpretation of the results, the comparisons between the two solutions are reported expressing the temperature as

$$
T^{*}=\frac{T-T_{c}}{T_{0}-T_{c}}
$$


where $T_{0}$ is the total temperature at the entry of LOS1, and $T_{c}$ the total temperature of the coolant entering CAV1.

The temperature data used as reference are from a thermal model (proprietary software SC03) based on correlations and calibrated in order to match thermocouples data in a set of selected points. The SC03 data is regarded as an overall, reasonably accurate indication of the thermal response, but strictly speaking, the values reported cannot be considered as experimental measurements, especially if looking in the details of the solution.

At the points located on the inner surfaces of the rotor cavity, namely FC1, FC2 and FC3, the coupled solution stabilizes after 10 thousand multigrid cycles (Fig. 23). In the outer part (point FC1), the temperature is approximately 10\% larger than in the SC03 solution. The difference is slightly larger at inner radii (point FC2) and in the labyrinth (point FC3).

Comparisons for the outer surface of the rotor cavity are shown in Fig. 24. The two solutions at IC1 and IC2 are very close $(<5 \%)$, whereas at IC3 the value provided by JM76 becomes $13 \%$ lower. It should be noted the slow convergence observed at point IC1, in a region of the inner cavity where there is very little recirculation and the heat flux is weak (see Fig. 16).

The results for the stator disc are shown in Fig. 25. Even in this case, the convergence rate for the lower part of the disc is relatively smaller. As the metal conduction propagates heat towards the lower part of the component, heat is extracted from the colder fluid; temperature contours of Figure 15 show a gradual change of colours near the disc, indicating very little convection in the front cavity. Hence, the thermal interaction between the disc and the part of coolant moving through the front rim seal, occurs on a time scale which is primarily controlled by turbulent diffusion.

In the SC03 solution, points SD1 and SD2 are at the same temperature, whereas in the coupled solution a strong temperature gradient develops on the outer disc surface. In this regard, the coupled solution is more convincing than that of SC03, because the presence of coolant entering the annulus must cool down the surface, as clearly visible from the metal temperature shown in Fig. 21. Moving at inner radii along the disc, point SD3, the difference between the two solutions widens significantly. According to the SC03 solution, there is little thermal difference throughout the disc, and the value at SD3 remains 
very close to that of SD1 and SD2. In the computation, the disc foot remains considerably colder, and SD3 is the point with the worse agreement.

This behaviour is believed to be a consequence of the assumptions in the model. The mixing planes between rotor and stator, in fact, average the flow and, therefore, the circumferential pressure variations driving ingestion are not modelled. The SC03 model otherwise is calibrated to account for the correct amount of hot gas that penetrates the cavity. This flow moves inwards along the disc, merges with the coolant, and leaves the cavity moving through the rotating wall. An estimate of the ingested flow obtained through integration of the velocity field indicates an underprediction of about $85 \%$ as compared to the value specified in the SC03 model.

\section{Heterogeneous set-up}

The simulation described above performed 60 thousand multigrid iterations, and lasted approximately nine days running on 13 nodes of a PC cluster, each node consisting of 16 Intel Xeon cores.

The aim of this section is to show that a significant speed-up can be achieved by appropriate selection of the numerical setting in each Hydra model. For this purpose, we consider the performance of three distinct computations, T1, T2 and T3, defined as reported in Tables 4 and 5. The benchmark computation presented in previous subsection (labelled as T1) adopts an identical numerical method for all models, consisting of 3-level multigrid cycles, with each cycle performing two RK5 iterations on each of the 3 meshes. This is designated as 2-2-2 cycle. The distribution of computational resources among the Hydra models is given in Table 4. In order to guarantee load balancing, the number of computing cores is set to be proportional to the workload of each individual model. In this respect, computation T1 behaves like those obtained using a monolithic code equipped with a standard partitioning algorithm.

Simulation T2 is an example of a different approach, in which the modularity of JM76 is exploited to concentrate computational resources for those models that introduce physical stiffness. In the low pressure turbine analyzed, the small convergence rate observed in the lower part of the disc (Fig. 25) derives from (1) slow heat conduction through the metal, and (2) small fluid convection in the front cavity. The time scale of both fluid and solid zones in the main annulus is significantly smaller, because the annulus flow is dominated by convection. Based on these considerations, in simulation T2 the multigrid cycle for zones 
LOS1, LOR1 and LOS2 is reduced from 2-2-2 to 1-1-1, and the corresponding numbers of computing cores is halved. The resulting surplus of resources is then allocated for those regions characterized by slow dynamics, namely the fluid zone CAV1 and both solid zones SOLID-DISC and SOLID-CAV. Since the exchange of data between Hydra Sessions and Coupler Units occurs before entering a multigrid cycle (cfr. Alg. 1), the workload of each individual Session can be preserved by maintaining constant the ratio between the number of cores and the number of iterations per cycle.

Simulation T3 is an example of hybrid modelling, using both the explicit and implicit flow solvers available in Hydra. The implicit scheme is known to be more efficient for flows dominated by diffusion, and is therefore employed for the inner and outer cavity flows. The Richardson iterations of the implicit scheme can be calibrated to guarantee that no additional waiting time is introduced for synchronization. Hence, for the three computations examined here, the CPU time per cycle is essentially the same.

Figure 26 shows the evolution of metal temperature at the points SD1, SD3 and IC1. It can be seen a much faster response obtained with simulations T2 and T3. The best performance is obtained in simulation T3. In this case, convergence for all points is reached after 20 thousand cycles. This corresponds to a reduction in the number of cycles by a factor of 3 , when compared to the reference computation T1. Simulations T2 and T3 give a very similar response for the points on the disc (SD1 and SD3), but at point IC2 the convergence rate remains significantly smaller for T2. Note that T2 and T3 employ the same settings for the solid regions. Hence, the different response observed at point IC2 has to be related to different convergence rates of the flow field. This effect can be appreciated in Fig. 27, where the convergence histories for the inner cavity flows are compared. The residual norm in T3 drops by 2 orders of magnitudes after 10 thousand cycles. In order to achieve the same reduction, T1 and T2 require approximately 30 thousand cycles.

Both the solver settings and loading combinations tested are clearly not optimal; these are presented here as examples to showcase the benefits and flexibility of the code coupling approach. Not surprisingly, different combinations can indeed produce the opposite effect and lead to convergence slow-down, or even instability in case of excessive decoupling. A dynamic and physically driven procedure is therefore required to exploit the potential of the method for complex multi-scale simulations: this development, 
including testing for unsteady effects and heterogeneous hardware, is part of on-going research.

\section{CONCLUSIONS}

The paper has described the application of a coupling methodology for the conjugate heat transfer analysis of secondary air systems. The method has been first validated using the test rig of a compressor cavity, and then applied for the analysis of an inter-stage cavity in a low pressure turbine assembly. The simulations have been carried using steady RANS. The resulting metal temperatures for the low pressure turbine are therefore underpredicted due to the lack of pressure driven ingestion.

The work presented is part of a program aimed at addressing multi-scale aero-thermal simulations. In this context, the methodology developed offers a series of advantages, summarised as follows:

Flexibility. The assembly is divided into several interconnected subdomains, in which different fluid and/or solid models are specified. Preprocessing for the various subdomains is performed separately, and this step is further simplified by the use of Chimera techniques that facilitate meshing and, more in general, relax the geometric constraints existing between two sub-domains.

Computing performance. The underlying concept of the method is inherently parallel and removes the main barriers for scalability that are typical of a monolithic approach both in the pre-processing and running, as well as in the post-processing. The system is composed of a series of sessions, behaving like the parallel processes of a monolithic code. The cost of the interface exchange is managed by an overlapping Coupler Unit process with dedicated memory and computational resources.

Potential for multi-fidelity capability. The system represents a natural framework for hybrid modelling, and paves the way to non-standard methodologies to be embedded selectively inside the simulation of complex systems.

The results discussed in the paper have primarily been focused to the logic and flexibility of the method for realistic applications. The paper also describes the features available to optimise the computational effort by using different and more efficient setting in each domain. This represents an important asset when looking at a widening range of features that coexist in a coupled system; examples are mesh size/topology, physical models (different gas-properties, fluid vs. solid, RANS vs. LES etc...), as well as 
numerical model (e.g. explicit vs. implicit scheme) or flow regimes (low speed vs. high speed). In this regard, given the increasing capability to include complexity, future work should address the ability to optimize the computational effort through automatic or semi-automatic allocation of resources, specific settings for robustness of physical interfaces, and efficient balancing of ranks between different sessions.

\section{ACKNOWLEDGMENT}

The current work has been developed within the Rolls-Royce project "GEMinIDS" WP4.1 Aerosimulation under the UK ATI research contract 113088. The authors also wish to thank Rolls-Royce plc for the permission to publish as well as colleagues for the engine model provided and the technical support received. 


\section{References}

[1] Young C., Smout, P.D., 2006. "Recent Advances in the Simulation of Gas Turbine Secondary Air Systems", Proceedings of the $25^{\text {th }}$ International Congress of the Aeronautical Sciences, Hamburg, Germany, September 3-8, 2006.

[2] Ho K.S., Urwiller C., Konan S.M., Liu J.S., Anguilar B., 2014. "Conjugate Heat Transfer Analysis for Gas Turbine Cooled Blade", Proceedings of ASME Turbo Expo 2014, Düsseldorf, Germany, June 16-20, 2014, ASME Paper No. GT2014-25952.

[3] Insinna M., Griffini D., Salvadori S., Martelli F., 2014. "Conjugate Heat Transfer Analysis of a Film Cooled High-Pressure Vane Under Realistic Combustor Exit Flow Conditions" Proceedings of ASME Turbo Expo 2014, Düsseldorf, Germany, June 16-20, 2014, ASME paper No. GT2014-25280.

[4] Illingworth J.B., Hills N.J., Barnes C.J., 2005. "3D Fluid-Solid Heat Transfer Coupling of an Aero Engine Pre-Swirl System", Proceedings of ASME Turbo Expo 2005, Reno, Nevada, USA, June 6-9, 2005, ASME paper No. GT2005-68939.

[5] Sun Z., Chew J.W., Hills N.J, Volkov K., Barnes C.J., 2010. "Efficient Finite Element Analysis/Computationa Fluid Dynamics Thermal Coupling For Engineering Applications", ASME J Turbomach, 132, pp. 1-9.

[6] Okita Y., Yamawaki S., 2002. "Conjugate Heat Transfer Analysis of Turbine Rotor-Stator Systems", Proceedings of ASME Turbo Expo 2002, Amsterdam, The Netherlands, June 3-6, 2002, ASME paper No. GT2002-30615.

[7] Dixon J.A., Valencia A.G., Coren D., Eastwood D., Long C., 2014. "Main Annulus Gas Path Interactions - Turbine Stator Well Heat Transfer", ASME J Turbomach, 136, pp. 1-16.

[8] Javiya U., Chew J.W., Hills N.J., 2015. "Coupled FE-CFD Thermal Analysis for a Cooled Turbine Disk", Proc IMechE Part C: J Mechanical Engineering Science, 0, pp. 1-16.

[9] He L., Oldfield M.L.G., 2011. "Unsteady Conjugate Heat Transfer", ASME J Turbomach, 133, pp. $1-12$. 
[10] Okita Y., 2006. "Transient Thermal and Flow Field in a Turbine Disk Rotor-Stator System", Proceedings of ASME Turbo Expo 2006, Barcelona, Spain, May 8-11, 2006. ASME paper No. GT200690033.

[11] Ganine V., Amirante D., Hills N.J., 2016. "Aero-Thermo-Mechanical Modelling and Validation of Transient Effects in a High Pressure Turbine Internal Air System", Proceedings of ASME Turbo Expo 2016, Seoul, South Korea, June 13-17, 2016. ASME Paper No. GT2016-57739.

[12] Horwood J.T.M., Hualca F.P., Wilson M., Scobie J.A., Sangan C.M., Lock G.D., 2018. "Unsteady Computation of Ingress Through Turbine Rim Seals", Proceedings of ASME Turbo Expo 2018, Oslo, Norway, June 11-15, 2018. ASME Paper No. GT2018-75321.

[13] Robak C.W., Faghri A., Thole K.A., 2019. "Analysis of Gas Turbine Rim Cavity Ingestion with Axial Purge Flow Injection", Proceedings of ASME Turbo Expo 2019, Phoenix, Arizona, USA, June 17-21, 2019. ASME Paper No. GT2019-91807.

[14] Owen M.J., Long C.A., 2015. "Review of Buoyancy-Induced Flow in Rotating Cavities", ASME J Turbomach, 137, pp. 1-13.

[15] Amirante D., Sun Z., Chew J.W., Hills N.J., 2016. "Modeling of Compressor Drum Cavities With Radial Inflow", Proceedings of ASME Turbo Expo 2016, Seoul, South Korea, June 13-17, 2016. ASME Paper No. GT2016-56505.

[16] Duchaine F., Jauré S., Poitou D., Quémerais E., Staffelbach G., Morel T., Gicquel L., 2015. "Analysis of High Performance Conjugate Heat Transfer with the OpenPALM Coupler", Comput. Sci. Disc., 8, pp. 1-19.

[17] Oh T.K., Tafti D., Nagendra K., 2019. "LES-Conjugate Heat Transfer Analysis of a Ribbed Cooling Passage Using the Immersed Boundary Method", Proceedings of ASME Turbo Expo 2019, Phoenix, Arizona, USA, June 17-21, 2019. ASME Paper No. GT2019-90397. 
[18] Slotnick J., Khodadoust A., Alonso J., Darmofal D., Gropp W., Lurie E., Mavriplis D., 2014. "CFD Vision 2030 Study: A Path to Revolutionary Computational Aeroscience", NASA Report No. NASA/CR2014-218178.

[19] Borgdorff J., Falcone J.L., Lorenz E., Bona-Casas C., Chopard B., Hoekstra A., 2013. "Foundations of Distributed Multiscale Computing: Formalization, Specification, and Analysis", J. Parallel Distrib. Comput., 73, pp. 465-483.

[20] Moinier P., 1999. "Algorithm Developments for an Unstructured Viscous Flow Solver", PhD dissertation, Oxford University, Oxford, UK.

[21] Hirsch C., 1990. "Numerical Computation of Internal and External Flows", John Wiley and Sons, New York.

[22] Misev C., Hills N.J., 2018. "Steepest Descent Optimisation of Runge-Kutta Coefficients for Second Order Implicit Finite Volume CFD codes", J. Comp. Phys., 354, pp. 576-592.

[23] Swanson R.C., Turkel E., Rossow C.C., 2007. "Convergence Acceleration for Runge-Kutta Schemes for solving the Navier-Stokes Equations", J. Comp. Phys., 224, pp. 365-388.

[24] Romagnosi L., Li Y., Mezine M., Teixeira M., Vilmin S., Anker J.E., Claramunt K., Baux Y., Hirsch C., 2019. "A Methodology for Steady and Unsteady Full-Engine Simulations", Proceedings of ASME Turbo Expo 2019, Phoenix, Arizona, USA, June 17-21, 2019. ASME paper No. GT2019-90110.

[25] Alonso J.J., Hahn S., Ham F., Herrmann M., Iaccarino G., Kalitzin G., LeGresley P., Mattsson K., Medic G., Moin P., Pitsch H., Schlüter J., Svärd M., Van der Weide E., You D., Wu X., 2006. "CHIMPS: A High-Performance Scalable Module for Multi-Physics Simulations", Proceedings of $42^{\text {nd }}$ AIAA/ASME/SAE/ASEE Joint Propulsion Conference \& Exhibit 2006, Sacramento, California, USA, July 9-12, 2006.

[26] Mainel M., Gunnar Ó.E., 2010. "The FlowSimulator Framework for Massively Parallel CFD Applications", Proceedings of PARA 2010: Conference on state of the Art in Scientific and Parallel Computing Reykjavik, Iceland, June 6-9, 2010. 


\section{Bibliography}

[27] Bungartz H.J., Lindner F., Gatzhammer B., Mehl M., Scheufele K., Shukaev A., Uekermann B., 2016. "preCISE - A Fully Parallel Library for Multi-Physics Surface Coupling", Comput. Fluids., 141, pp. 250-258.

[28] Tang Y.H., Kudo S., Bian X., Li Z., Karniadakis G., 2016. "Multiscale Universal Interface: a Concurrent Framework for Coupling Heterogeneous Solvers", J. Comput. Phys., 297, pages 13-31.

[29] Atkins N.R., 2013. "Investigation of a Radial-Inflow Bleed as a Potential for Compressor Clearance Control", Proceedings of ASME Turbo Expo 2013, San Antonio, Texas, USA, June 3-7, 2013. ASME Paper No. GT2013-95768.

[30] De Laborderie J., Duchaine F., Gicquel L., Vermorel O., Wang G., Moreau S., 2018. "Numerical Analysis of High-Order Unstructured Overset Grid Method for Compressible LES of Turbomachinery", J. Comput. Phys., 363, pp. 371-398.

[31] Meakin R.L., 2001. "Object X-Rays for Cutting Holes in Composite Overset Structured Grids", Proceedings of $15^{\text {th }}$ AIAA Computational Fluid Dynamics Conference, Anaheim, California, USA, June 11-14, 2001.

[32] Poncet S., Chauve M.P., Schiestel R., 2005. "Batchelor versus Stewartson Flow Structures in a Rotor-Stator Cavity with Throughflow" Phys. Fluids, 17, pp. 1-15. 


\begin{tabular}{ccccccc}
\hline \hline $\begin{array}{c}\Omega \\
{[\mathrm{rad} / \mathrm{s}]}\end{array}$ & $\begin{array}{c}T_{\text {rad } 2} \\
{[\mathrm{~K}]}\end{array}$ & $\begin{array}{c}T_{\text {rad3 } 3} \\
{[\mathrm{~K}]}\end{array}$ & $\begin{array}{c}T_{\text {inlet }} \\
{[\mathrm{K}]}\end{array}$ & $\begin{array}{c}\operatorname{Re}_{z} \\
\times 10^{-5}\end{array}$ & $\begin{array}{c}\operatorname{Re}_{\theta} \\
\times 10^{-6}\end{array}$ & $\dot{m}_{\text {rad }} / \dot{m}_{\text {bore }}$ \\
\hline 730 & 381 & 383 & 340 & 1.5 & 8.5 & $4.4 \%$ \\
\hline
\end{tabular}

Table 1: Running conditions of the test rig

\begin{tabular}{ccccccccccc}
\hline \hline LOS1 & LOR1 & LOS2 & CAV1 & BL-DISC & CAV2 & BL-CAV2 & SOLID & SOLID & SOLID & SOLID \\
DISC
\end{tabular}

Table 2: Mesh size of each domain (million nodes)

\begin{tabular}{cccccc}
\hline \hline$\dot{m}_{i}$ & $\dot{m}_{i}^{S 2}$ & $\dot{m}_{o}^{S 2}$ & $\dot{m}_{f r s}$ & $\dot{m}_{r r s}$ & $\dot{m}_{c}$ \\
\hline 1.00 & 0.99391 & 1.00239 & 0.00158 & 0.00708 & 0.00860 \\
\hline
\end{tabular}

Table 3: Predicted mass flow rate at various interfaces 


\begin{tabular}{cccccccccccc}
\hline \hline & LOS1 & LOR1 & LOS2 & CAV1 & $\begin{array}{c}\text { BL- } \\
\text { DISC }\end{array}$ & CAV2 & BL- & SOLID & SOLID & SOLID & SOLID \\
& & & & & & CAV2 & LOS2 & LOR 1 & CAV & DISC \\
\hline T1 & 3 & 35 & 35 & 42 & 20 & 26 & 20 & 5 & 6 & 1 & 1 \\
T2,T3 & 3 & 17 & 18 & 63 & 30 & 26 & 20 & 5 & 6 & 3 & 3 \\
\hline
\end{tabular}

Table 4: Number of computing cores allocated for each Hydra model in computation T1, T2 and T3

\begin{tabular}{rcccccccccccc}
\hline \hline & LOS1 & LOR1 & LOS2 & \multirow{2}{*}{ CAV1 } & BL- & CAV2 & BL- & SOLID & SOLID & SOLID & SOLID \\
& & & & & DISC & & CAV2 & LOS2 & LOR 1 & CAV & DISC \\
\hline T1 & $2-2-2$ & $2-2-2$ & $2-2-2$ & $2-2-2$ & $2-2-2$ & $2-2-2$ & $2-2-2$ & $2-2-2$ & $2-2-2$ & $2-2-2$ & $2-2-2$ \\
T2 & $1-1-1$ & $1-1-1$ & $1-1-1$ & $3-3-3$ & $2-2-2$ & $2-2-2$ & $2-2-2$ & $2-2-2$ & $2-2-2$ & $6-6-6$ & $6-6-6$ \\
T3 & $1-1-1$ & $1-1-1$ & $1-1-1$ & $i m p-6$ & $i m p-4$ & $i m p-4$ & $i m p-4$ & $2-2-2$ & $2-2-2$ & $6-6-6$ & $6-6-6$ \\
\hline
\end{tabular}

Table 5: Multigrid cycle definition for each Hydra model in computation T1, T2 and T3, and number of Richardson iterations defined for the implicit solvers adopted in T3.

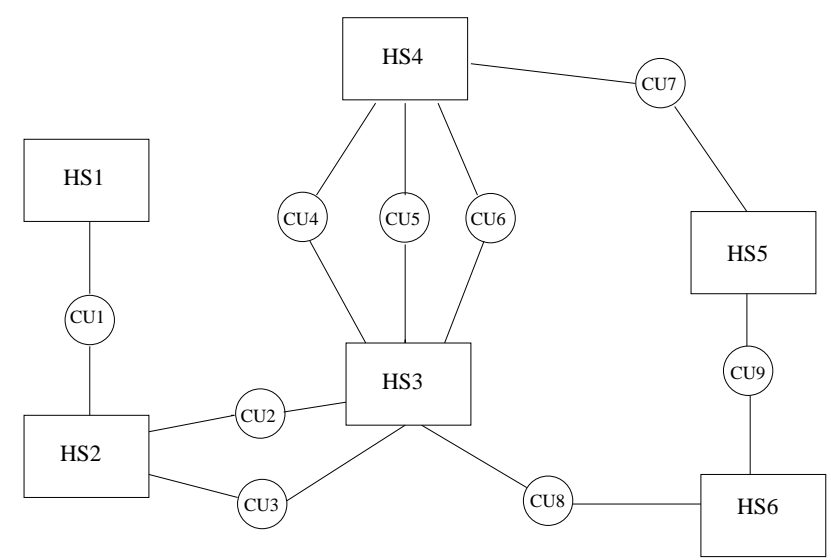

Figure 1: Schematic representation of a coupled model in JM76 

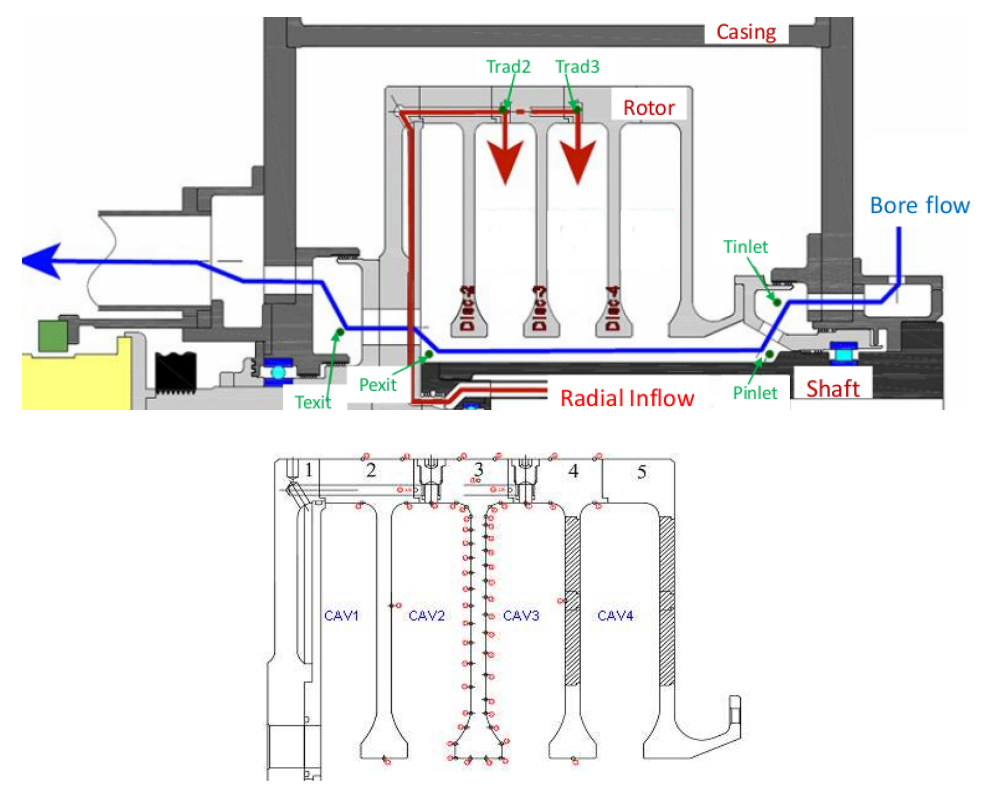

Figure 2: Validation test case. Experimental rig (top), and locations of thermocouples (bottom).

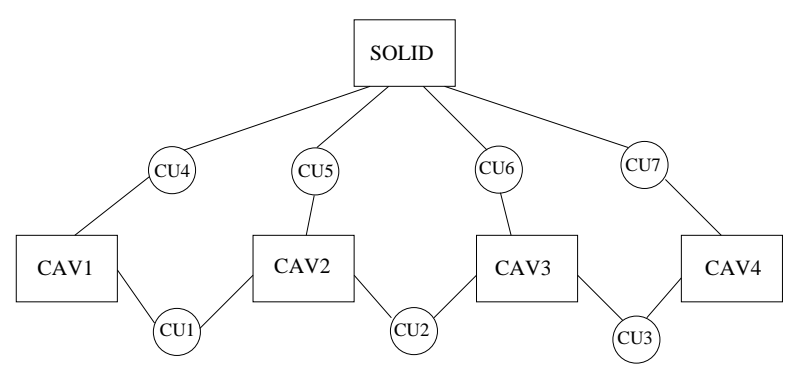

Figure 3: JM76 model adopted for the rig of Fig. 2

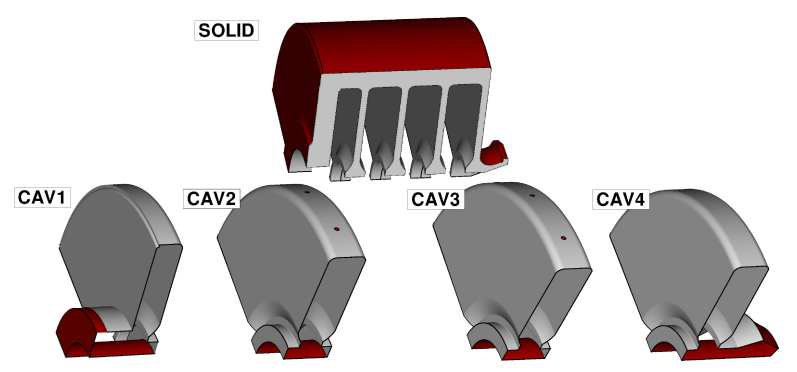

Figure 4: Computational domains for the rig of Fig. 2. Red surfaces are those left uncoupled during the simulation, i.e. those for inlet/outlet and walls with prescribed temperatures. 

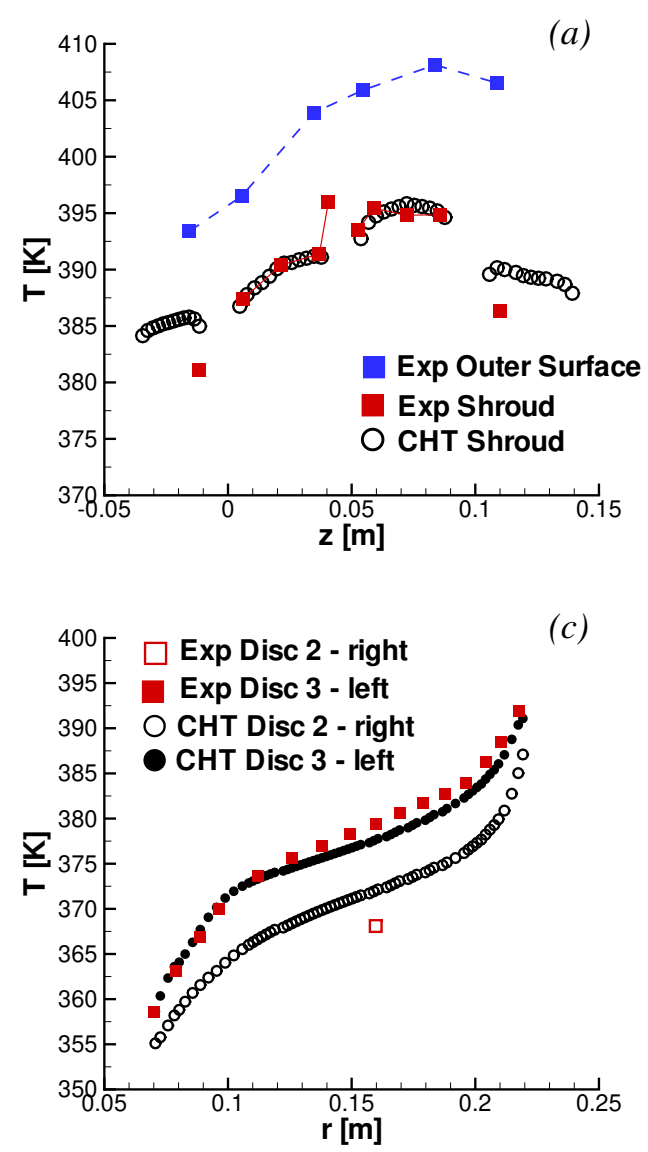
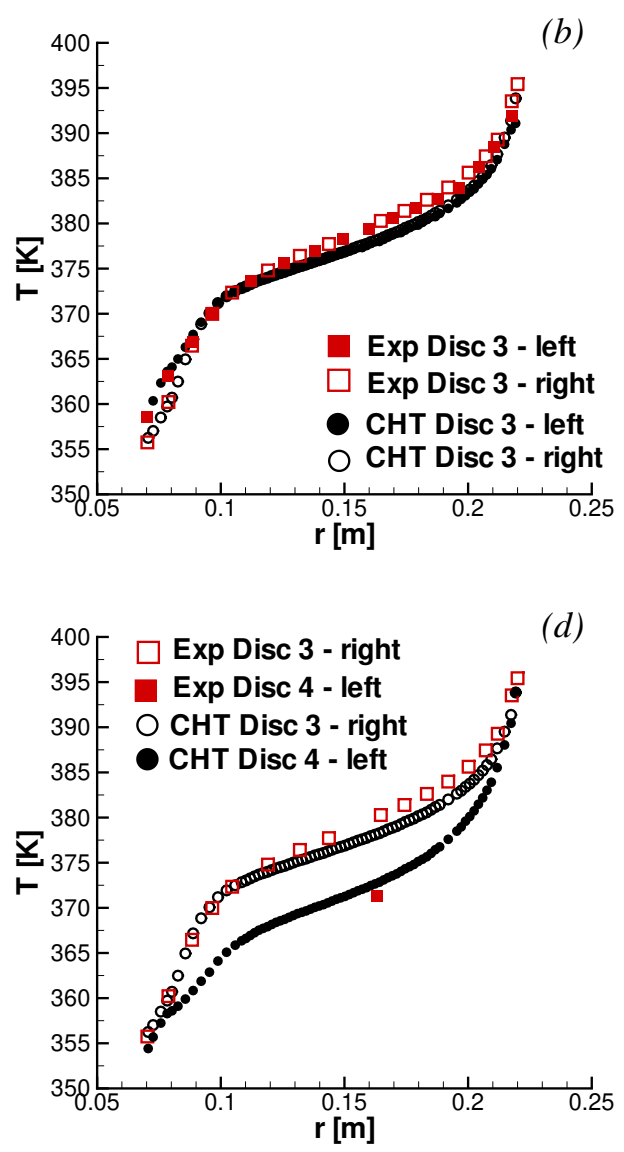

Figure 5: Validation test case. Metal temperature computed for the various components. $(a)$ : shroud; (b): both sides of DISC3; (c): both discs of CAV2; (d): both discs of CAV3.

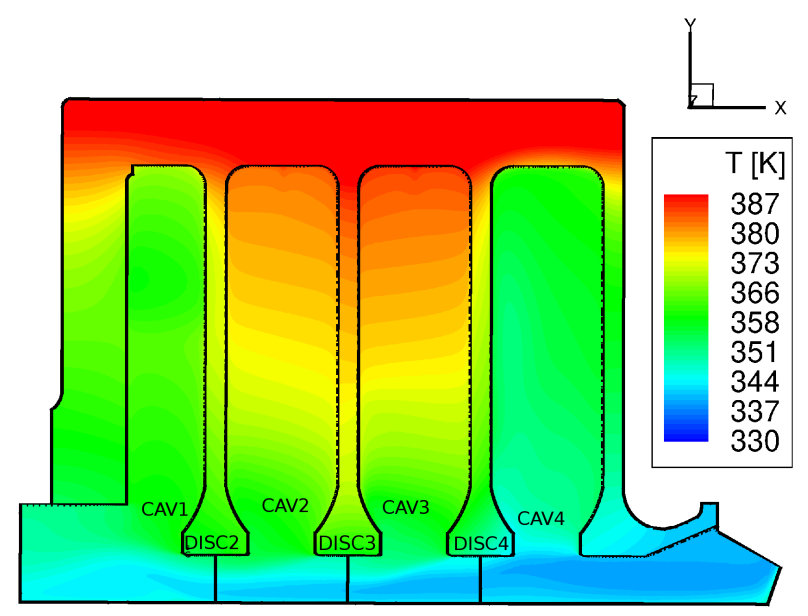

Figure 6: Validation test case. Temperature contours on the periodic surface of the coupled model. In the cavities equipped with radial inflow (CAV2 and CAV3) the fluid is approximately at the same temperature as the separating disc (DISC3). 


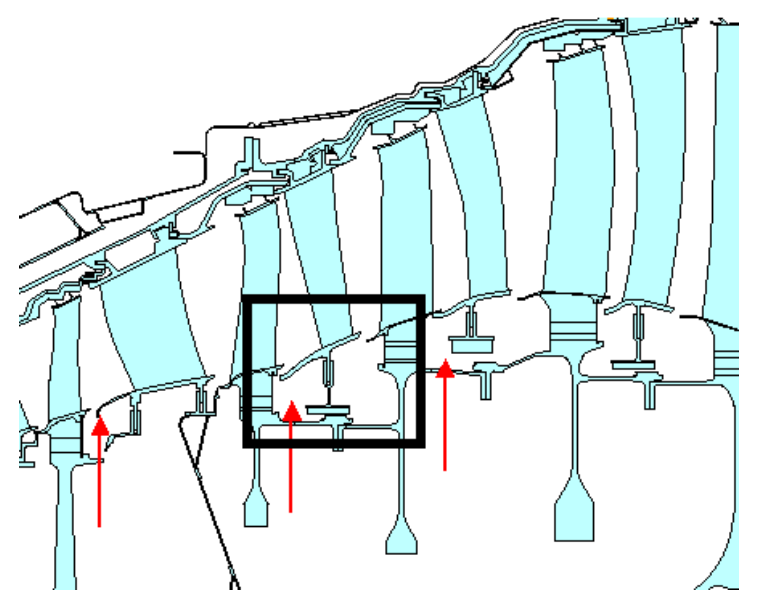

Figure 7: Low pressure turbine assembly. Enclosed in the square is the inter-stage cavity under investigation.

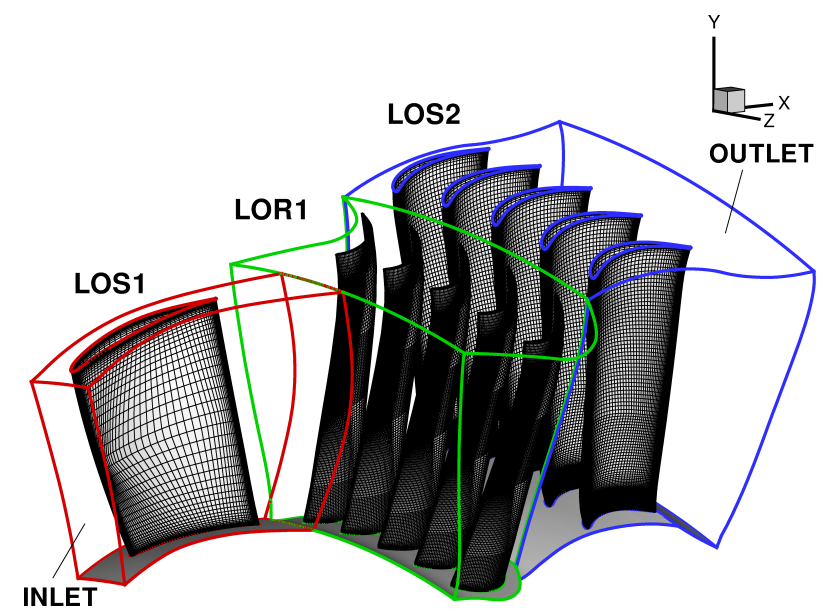

Figure 8: Fluid zones in the main annulus. The domain LOS2 includes the vanes attached to the stator disc framed in Fig. 7. 


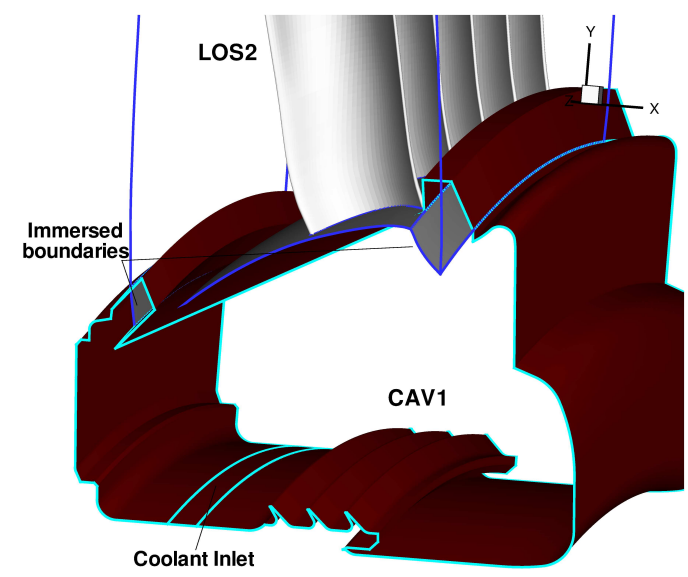

Figure 9: Fluid zone CAV1 overlapping with fluid zone LOS2. Two couples of immersed boundaries are located in the rim seals regions.

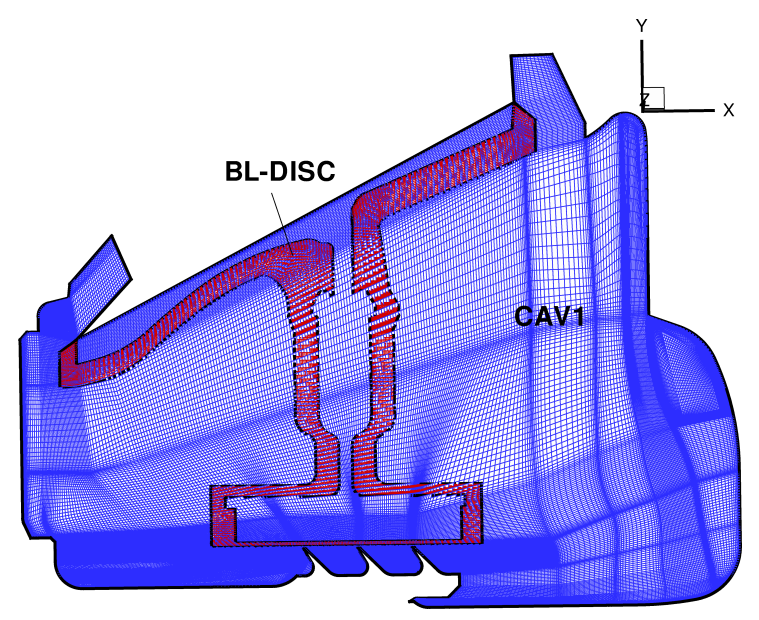

Figure 10: Overlapping grids adopted for the inter-stage cavity 


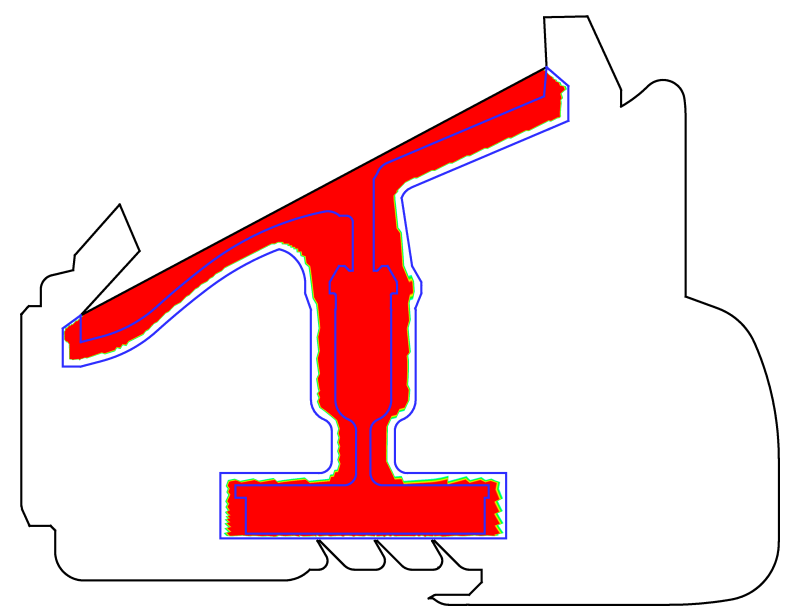

Figure 11: Hole region derived in fluid zone CAV1. The green line identifies the fringe nodes for CAV1. The blue line identifies the IMB nodes of BL-DISC.

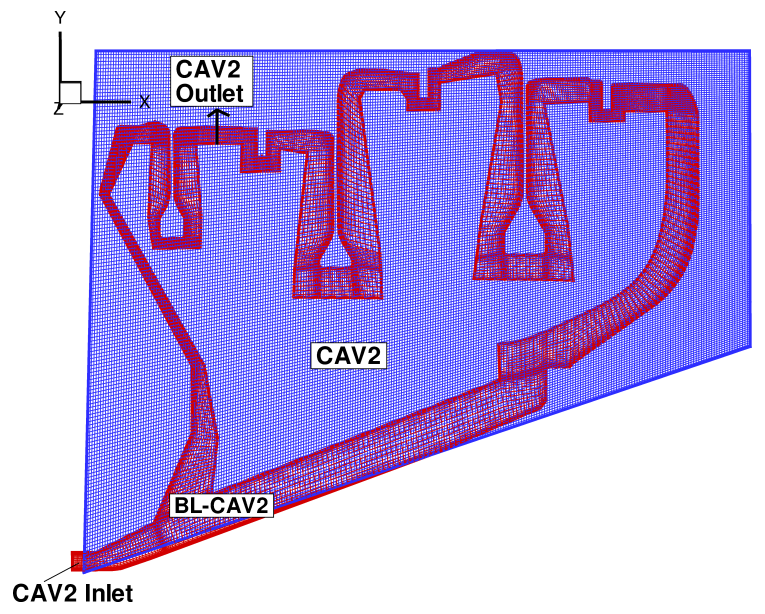

Figure 12: Overlapping grids used for the inner cavity 


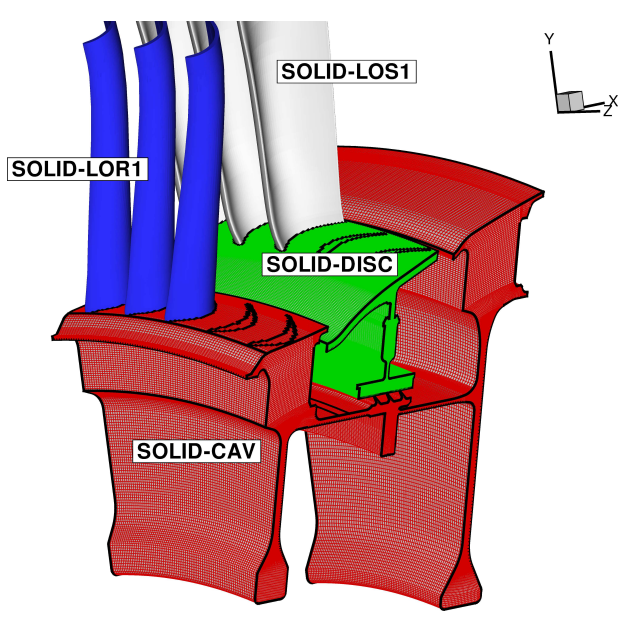

Figure 13: Solid models. For clarity, only 3 blades are reported for each bladerow. The traces on the outer surfaces of the disc and rotor cavity identify THC interfaces.

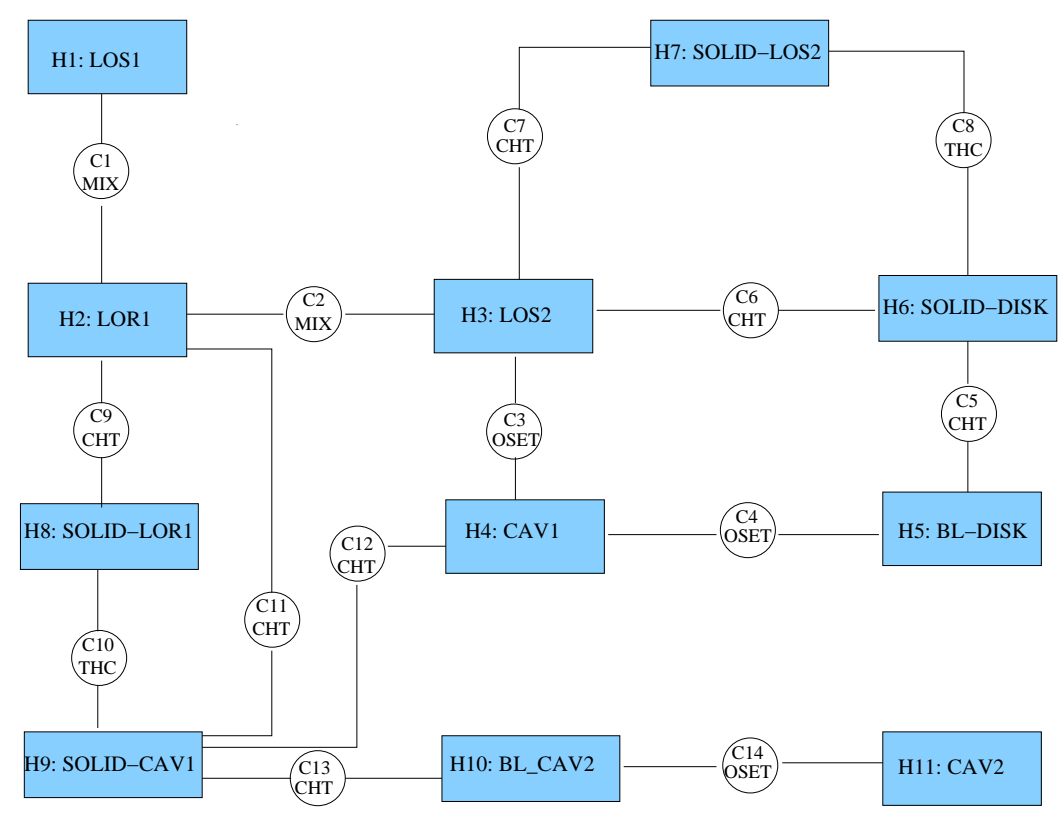

Figure 14: JM76 model adopted for the low pressure turbine 


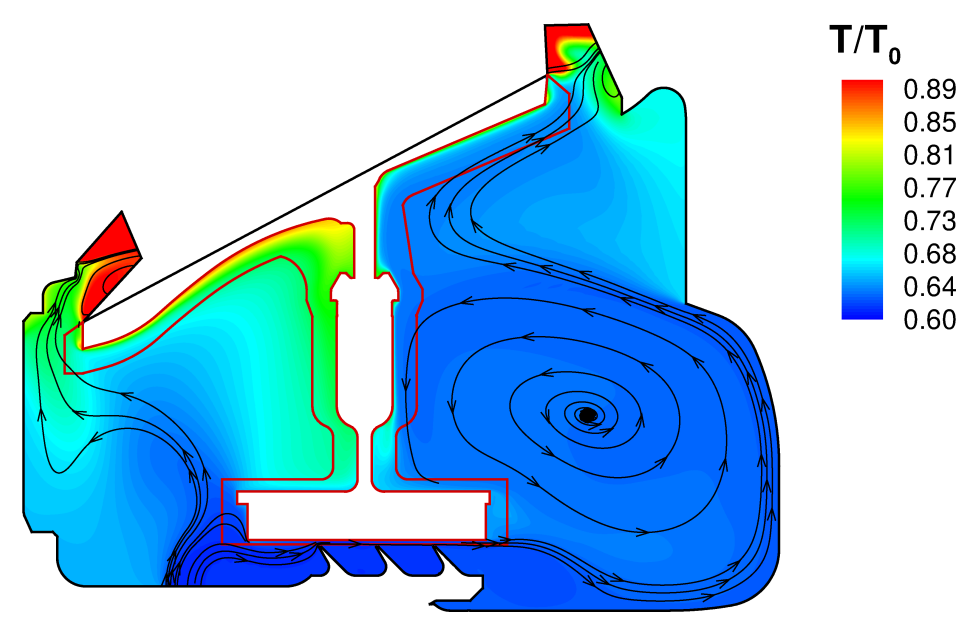

Figure 15: Fluid temperature in the stator well

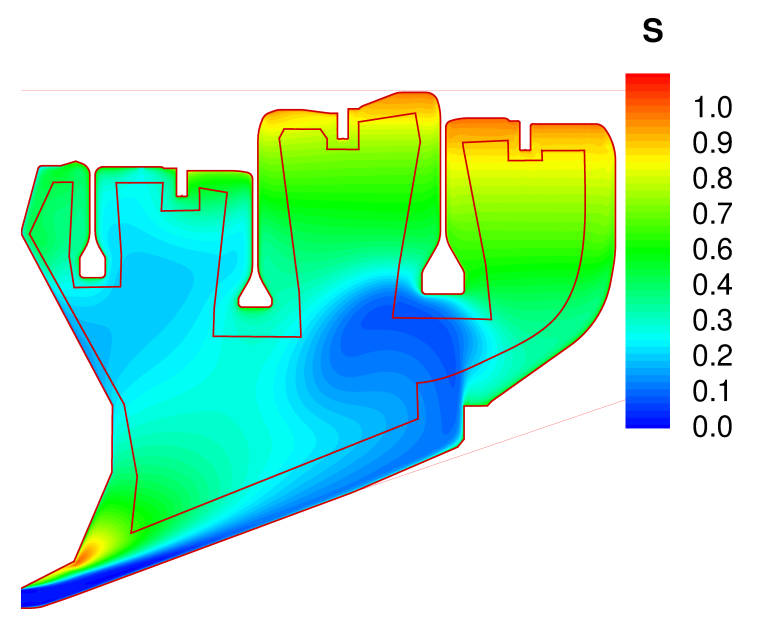

Figure 16: Swirl ratio in the inner cavity 


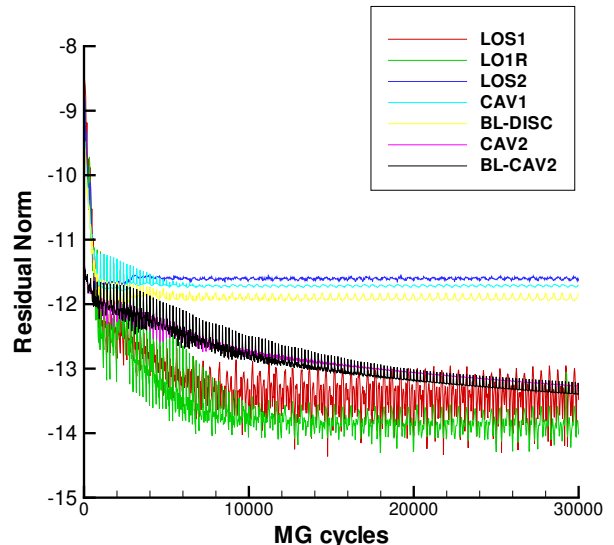

Figure 17: Convergence histories of the fluid models

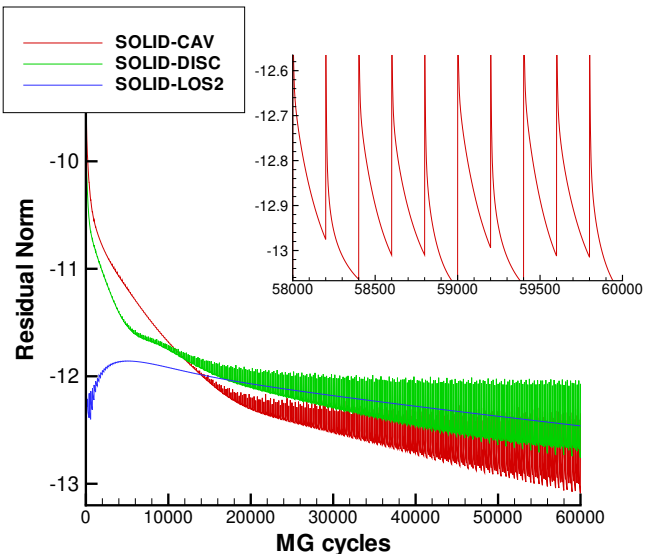

Figure 18: Convergence histories of the solid models 


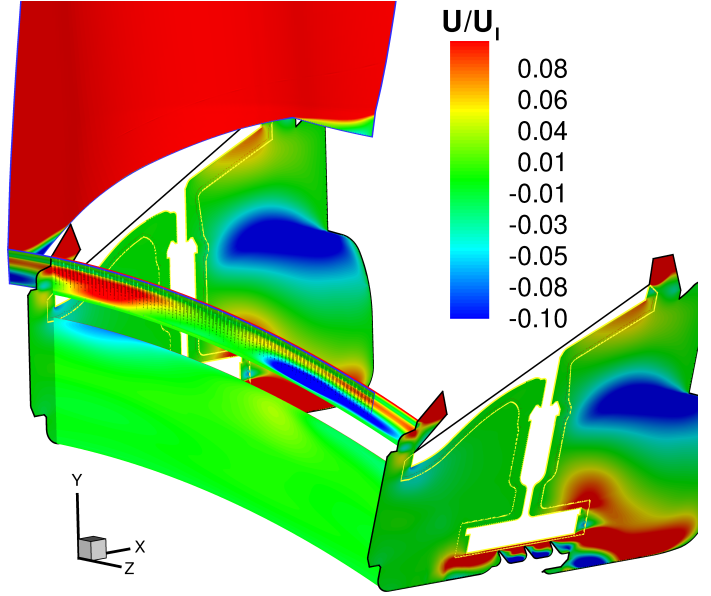

Figure 19: Axial velocity contours on a cross-section cutting the model CAV1 and overlapping with the immersed boundary of model LOS2

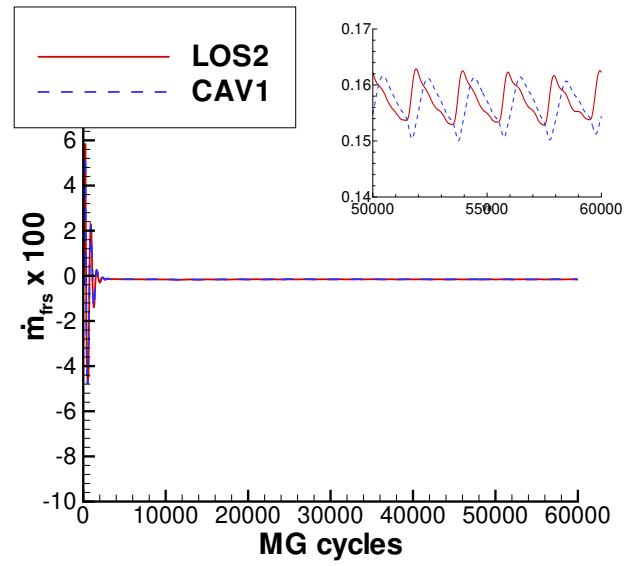

Figure 20: Mass flow rate through the front rim seal, measured from both models sharing the overset interface, namely LOS2 and CAV1 


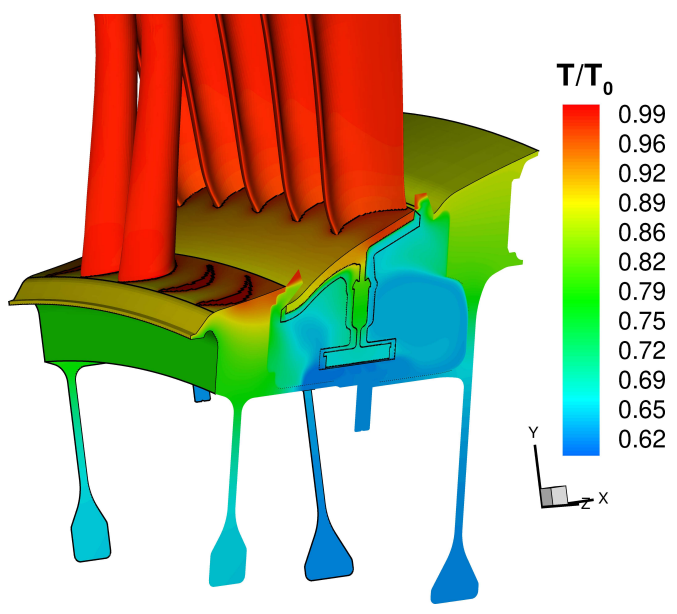

Figure 21: Three-dimensional view of the assembled model, with superposed fluid and solid temperatures

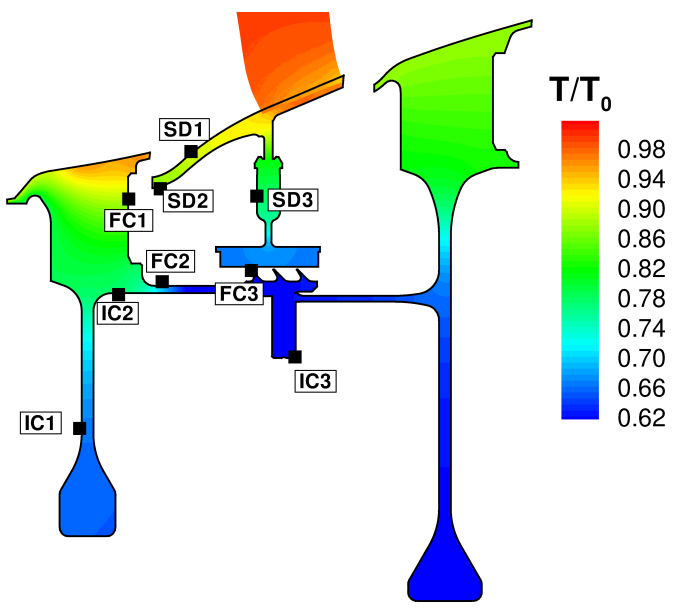

Figure 22: Metal temperature contours and control points location 


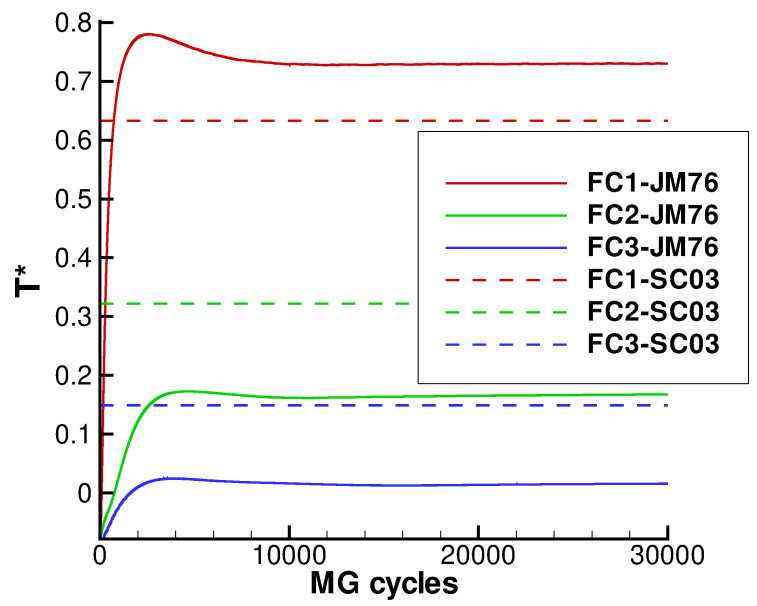

Figure 23: Metal temperature at control points on the inner surface of the inter-stage cavity

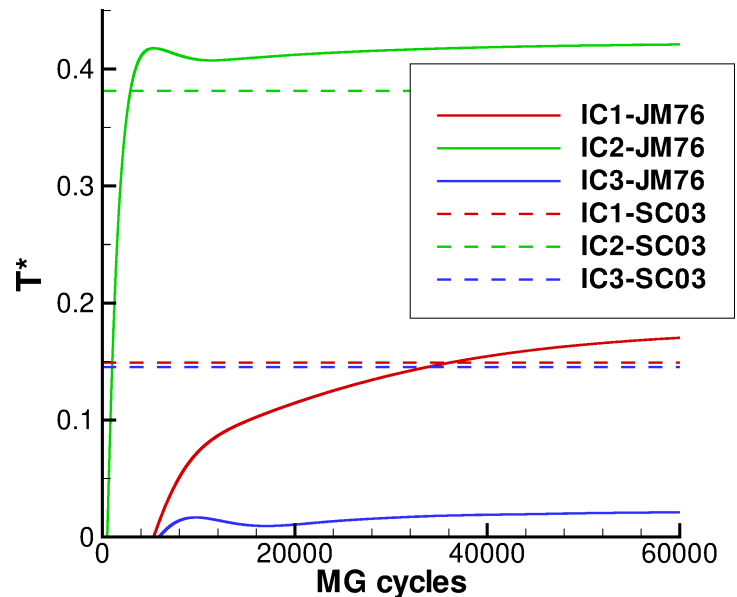

Figure 24: Metal temperature at control points on the outer surface of the inter-stage cavity 


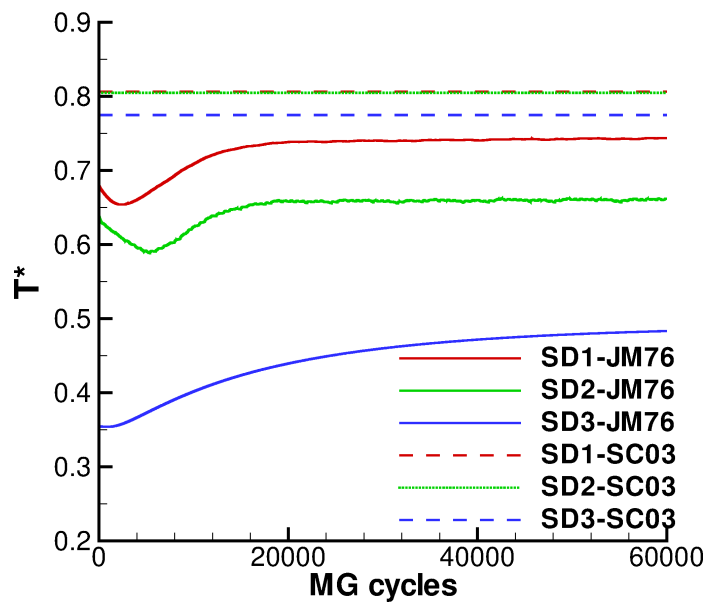

Figure 25: Metal temperature at control points along the disc

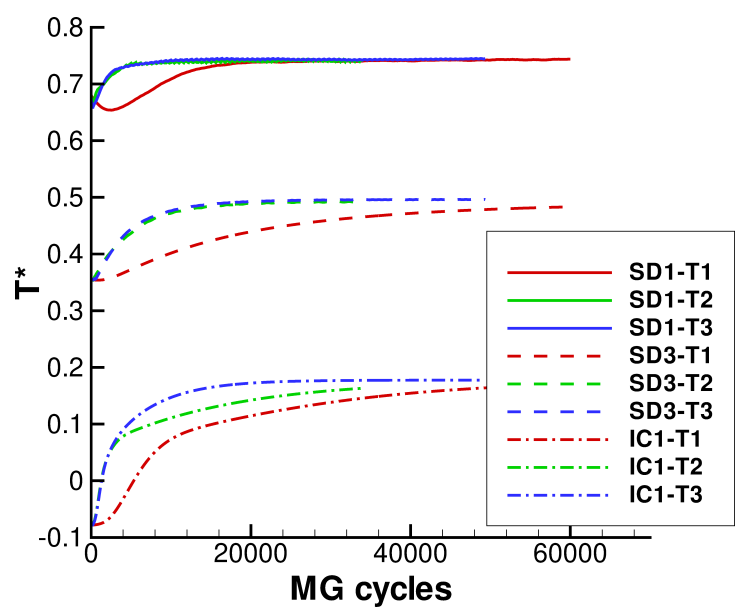

Figure 26: Performance of simulations T1, T2 and T3. Metal temperature at control points SD1, SD3 and IC1. 


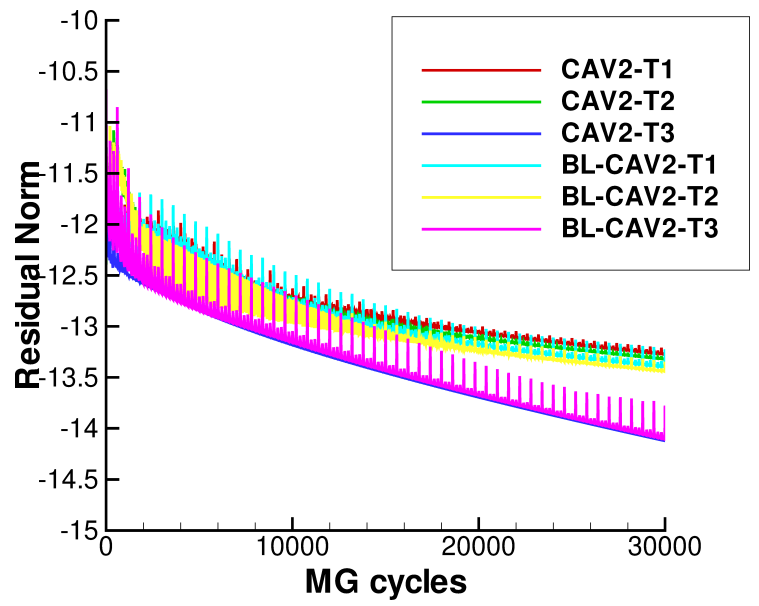

Figure 27: Performance of simulations T1, T2 and T3. Convergence histories for zones CAV2 and BLCAV2. 